\title{
Dark-rim-free ungated first-pass perfusion CMR with 3-Slice end-systolic imaging: initial experience
}

\author{
Behzad Sharif ${ }^{*}$, Reza Arsanjani ${ }^{1,2}$, Aryeh Shalev², Rohan Dharmakumar ${ }^{1}$, C Noel Bairey Merz², Daniel S Berman ${ }^{1,2}$, \\ Debiao $\mathrm{Li}^{1}$
}

From 17th Annual SCMR Scientific Sessions

New Orleans, LA, USA. 16-19 January 2014

\section{Background}

First-pass perfusion (FPP) cardiac MR (CMR) imaging has been shown to have a high performance for diagnosis of coronary artery disease (CAD). Reliability of FPP imaging, however, is hindered by dark-rim artifacts (DRAs) and the need for near-perfect ECG gating. The latter can be challenging in the presence of arrhythmias or heart-rate variations during stress. Moreover, endsystolic (ES) imaging has recently been shown to provide improved visualization of subendocardial defects [1]. We developed an innovative ungated FPP technique capable of simultaneously eliminating DRAs $[2,3]$ and enabling reconstruction of all slices at ES. We hypothesized that the developed method achieves DRA-free imaging and high accuracy in patients with suspected $\mathrm{CAD}$, using nuclear myocardial perfusion imaging (MPI) as the reference.

\section{Methods}

Based on the so-called "Ungated Cine FPP" approach [4], a multi-slice magnetization-driven [5-7] method was developed for ungated FPP imaging. Figure 1 highlights the main differences of the proposed pulse sequence compared with the conventional FPP method. All scans were performed on a $3 \mathrm{~T}$ clinical scanner using an ungated RF-spoiled GRE sequence with continuous golden-angle radial acquisition as in Figure 1b (flip angle $=21^{\circ}$, resolution: $1.7 \times 1.7 \times 10 \mathrm{~mm}$ ). The reconstruction method used automatic self-gating and optimally apodized [3] compressed sensing for DRA-free accelerated reconstruction. Normal subjects $(\mathrm{n}=6)$

${ }^{1}$ Biomedical Imaging Research Institute, Cedars-Sinai Medical Center, Los Angeles, California, USA

Full list of author information is available at the end of the article were studied using both the proposed and conventional methods. Patients $(n=9)$ with suspected CAD on the basis of recent abnormal SPECT/PET MPI underwent adenosine stress/rest FFP CMR. Three patients returned for a second study using the conventional method.

\section{Results}

The ungated FPP studies in normal subjects were all of high quality and demonstrated normal perfusion. A representative patient study is shown in Figure 2. Stressinduced hypoperfusion was observed in the ES Ungated Cine FPP images, corresponding to a reversible defect on PET MPI (Figure 2b). Figure 2c shows the mid slice for the conventional FPP scan. Based on nuclear MPI studies, sensitivity and specificity of the developed method were $93 \%$ and $95 \%$, respectively. The minor disagreements can be explained by the presence of subendocardial defects and possible artifacts on SPECT. All images were reviewed and no DRAs was detected on the Ungated Cine FPP images (2 readers, consensus). However, for the 3 studies using the conventional method, mild-moderate DRA was observed in $21 \%$ of the segments.

\section{Conclusions}

Conventional FPP methods are prone to DRAs, require accurate ECG gating, and do not provide the freedom to image all slices at ES. The developed method overcomes these challenges and is an attractive alternative with the advantage of simplicity (no gating), higher accuracy in the subendocardium (no DRAs, ES imaging), and thereby potentially improved reliability. Preliminary results in healthy volunteers and patients with suspected CAD were of high quality and showed high accuracy compared to nuclear MPI. 

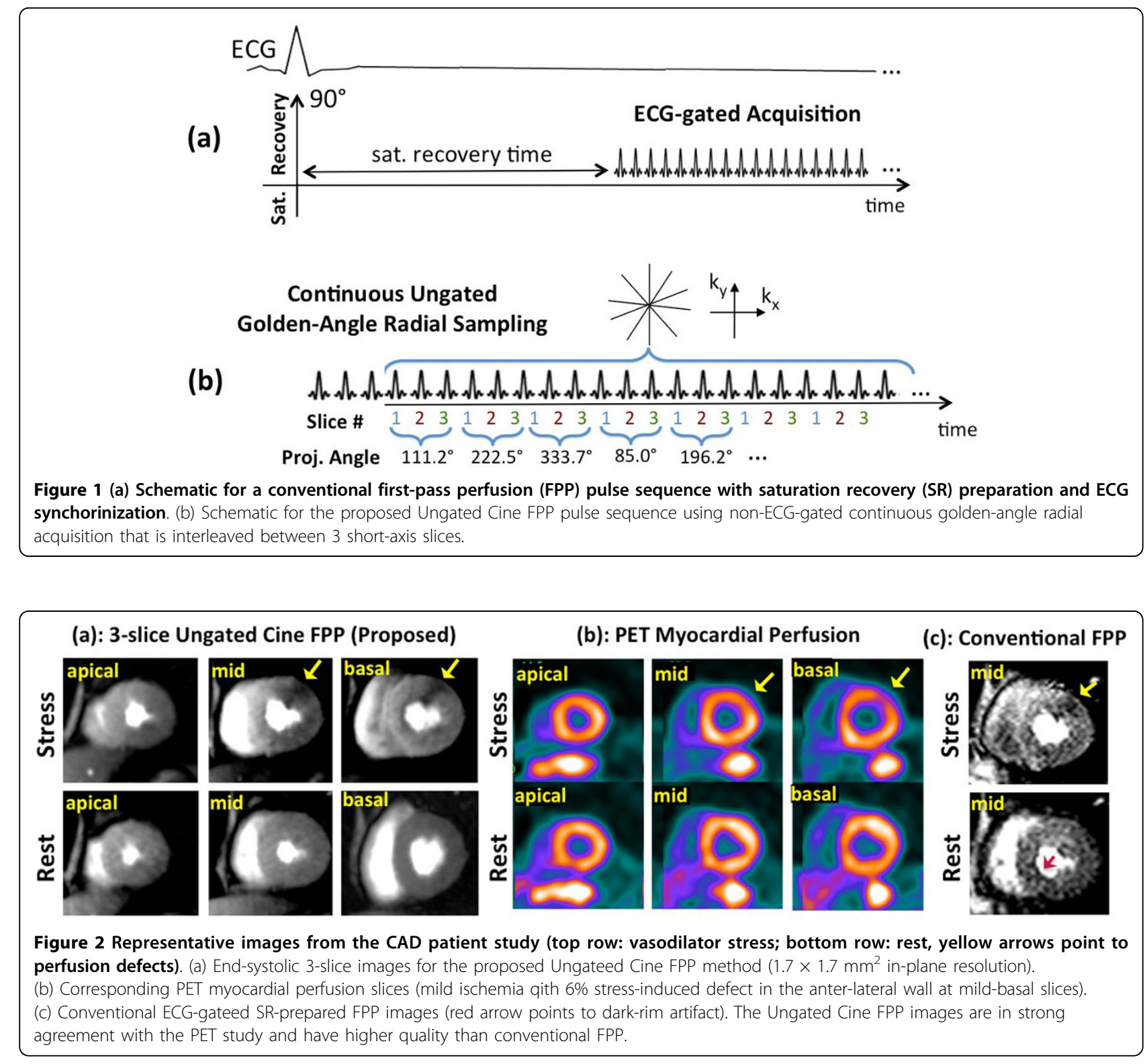

\section{Funding}

Grant sponsors: National Institutes of Health grants nos. NHLBI R01HL38698 and R01HL091989. American Heart Association Postdoctoral Fellowship Award 11POST7390063.

\section{Authors' details}

'Biomedical Imaging Research Institute, Cedars-Sinai Medical Center, Los Angeles, California, USA. ${ }^{2}$ Heart Institute, Cedars-Sinai Medical Center, Los Angeles, California, USA.

Published: 16 January 2014

\section{References}

1. Motwani, Plein, et al: Radiology 2012, 262.

2. Sharif, Li, et al: JCMR 2013, 15(Suppl 1):O3.
3. Sharif, Li, et al: MRM 2013, doi: 10.1002/mrm.24913.

4. Sharif, Li, et al: JCMR 2013, 15(Suppl 1):01.

5. Judd, et al: MRM 1995, 34

6. DiBella, et al: MRM 2012,67.

7. Giri, Simonetti: MRM 2013.

doi:10.1186/1532-429X-16-S1-P177

Cite this article as: Sharif et al:: Dark-rim-free ungated first-pass perfusion CMR with 3-Slice end-systolic imaging: initial experience.

Journal of Cardiovascular Magnetic Resonance 2014 16(Suppl 1):P177. 